\title{
Frequency of Dyslipidemia In Type 2 Diabetic Patients In Karachi
}

\author{
Sehrish Shafique, Daud Mirza, Summayya Shawana, Shahneela Tabassum, Naveed Faraz
}

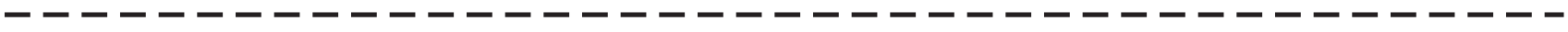

ABSTRACT:

Objective: To find out the frequency of dyslipidemias in type 2 diabetic patients.

Study Design and Setting: This was cross-sectional study and conducted in a different clinics in Karachi during 3 months period.

Methodology: Those patients who fulfilled the inclusion criteria and attended the OPD were selected. Patient were advised to bring their lipid profile report in next visit if not already done. The total dyslipidemias were presented by their frequencies and percentages with $95 \%$ confidence interval.

Results: Out of 383 patients with diabetes mellitus 210 (55\%) had dyslipidemia. Among which 76\% had elevated lowdensity lipoprotein (LDL), $66.6 \%$ had elevated serum cholesterol, 57\% patient had elevated triglyceride. p- value of 0.05 was taken as statistically significant.

Conclusion: It was concluded that increased frequency of dyslipidemias, with elevated low-density lipoprotein, cholesterol and triglycerides levels seen in diabetic patients.

Keyword: coronary heart disease, Diabetes mellitus, dyslipidemia, HbA1C.

\section{INTRODUCTION:}

Diabetes is one of those disease that is of high concerns because of its increasing number of cases. It is expected that number of diabetics will increase from 382 million in 2013 to 592 million by $2035^{1}$. According to International Diabetes Federation estimates in 2013,35 countries out of 219 have about $12 \%$ prevalence of diabetes. Among the Asian population $20 \%$ is currently affected due to the disease. In our country 7 million people suffer from hyperglycemia. The prevalence of the disease in our country is $18-46 \%$. The occurrence of diabetes with metabolic syndrome is 46$75 \%$ in Pakistan. ${ }^{2}$ It is one of the chronic metabolic diseases which is characterized by hyperglycemia, occurring due to defects in either insulin secretion, action, or both. ${ }^{3,4}$ Poorly controlled diabetes can result in many long-term complications which can lead to damage and dysfunction or failure of many vital organs, especially the eyes, kidneys,

Sehrish Shafique
Assistant Professor, Department of Medicine
Bahria University Medical and Dental College
I Email: drsehrish80@yahoo.com
I Daud Mirza
I HOD \& Associate Professor, Department of Oral pathology
I Bahria University Medical and Dental College
I Summayya Shawana
I Associate Professor, Department of Pathology
I Bahria University Medical and Dental College
I Shahneela Tabassum
I Bahriant Professor, Department of Neurology
I Naveed Faraz
I Professor, Department of Pathology
| Bahria University Medical and Dental College
| Received: 14-12-18
I Accepted: 26-02-19

nerves, heart, brain, blood vessels which are more affected along with increased risk of local and systemic infections in these patients. ${ }^{4}$ Because of developing these complications, the patients are also at a high risk of disability and premature death. ${ }^{4}$ Among these complications, Cardiovascular disease is of high concern in type 2 diabetics, as more than $60-70 \%$ die from coronary heart disease. ${ }^{5,6}$ According to American Heart Association, at $70 \%$ of the people with diabetes aged 65 or older die of diseases due to atherosclerotic changes in arteries supply heart and CNS. ${ }^{7}$ Diabetic patients have four times more chances to have coronary heart disease than non- diabetes. Thus diabetes is considered as equivalent to coronary artery disease. ${ }^{8}$

Dyslipidemia is common in diabetics..$^{6,7,8}$ In diabetic dyslipidemia there are lipid and lipoprotein abnormalities. It is characterized by elevated fasting and non-fasting triglycerides (TG) and TG-rich lipoproteins, e.g., chylomicrons and very-low-density lipoprotein (VLDL), low high-density lipoprotein cholesterol (HDL-C) and often also elevated low-density lipoprotein cholesterol (LDL-C) concentrations, increased small dense LDL particles. ${ }^{8,9}$ The pathophysiology behind these abnormalities is that they occur due to impaired VLDL secretion and there is decreased hepatic uptake of chylomicrons and their remnants also. ${ }^{8,9,10}$ Furthermore, lipolysis in these patients is also suppressed due to the presence of insulin resistance in adipose tissue which further contributes to diabetic dyslipidemia. So, there are number of factors which can contribute to deranged lipid markers from normal value in human body in diabetics, apart from insulin resistance, there is also deficiency of adipocytokines, which are also considered as the contributing factors in causing the alteration in lipid metabolism. ${ }^{8}$ The exact pathophysiology is still poorly understood, but it is seen that because of insulin resistance there is activation of 
intracellular lipases which in turn increase the release of no esterified fatty acids (NEFA) from the stored triglycerides in the adipose tissue and increases the hepatic triglyceride production which also causes increase in apolipoprotein B production. ${ }^{10,11}$ So, the normal inhibitory effect of insulin on hepatic apolipoprotein B production and triglyceride secretion in VLDL is lost, and the VLDL that is secreted is larger and more triglyceride-rich. There is also reduced VLDL catabolism which also increases the triglyceride levels. ${ }^{9,10}$ Lipoprotein lipase which is an enzyme located on vascular endothelium is responsible for the rate of removal of triglycerides from the circulation, but it is downregulated in diabetics due to insulin resistance or deficiency. ${ }^{10}$ This reduction in lipoprotein lipase activity further also contributes to deranged lipid markers from normal value in human body.

Recent studies suggest that low HDL cholesterol is also an independent factor not only for causing cardiovascular disease but also for the development of diabetes itself. ${ }^{12,13,14,15}$ In diabetic patients, improvement in the glycemic control not only cause reduction in cholesterol and triglyceride levels but also increases the catabolism of LDL through the upregulation of its receptors and reduced glycation. ${ }^{14,16}$ The rationale of our study was to find out the frequency of deranged lipid markers from normal value in patients with diabetes as earlier diagnosis of such abnormalities in diabetics can minimize and prevent the mortality due to coronary artery disease. Also, both of these diseases are commonly prevalent in our society and their mortality increase many times when they occur simultaneously.

\section{METHODOLOGY:}

The study was conducted in a private clinic in Karachi for a period of three months. All diagnosed cases of diabetes mellitus for more than 3 years with $\mathrm{Hb} \mathrm{AlC}$ of $7 \%$ or more, irrespective of age, gender were included and the patients suffering from cancer, chronic liver disease, hypothyroidism, patients on lipid lowering drugs and chronic renal disease were excluded from the study. The ethical approval was taken from the ethical review committee. Consent was taken before collection of data and also explaining the significance of this study and its procedure. Those patients who were selected after inclusion criteria and attending the OPD were selected. Patients were advised to bring their lipid profile report in next visit if not already done. The total deranged lipid markers from normal value in human body were presented in the form of frequencies and percentages with 95\% confidence interval and compared with standard NCEP chart given below. For data analysis SPSS version 20 were used. Different variables were calculated through frequency and percentage.

\section{RESULTS:}

In our study a total of 383 cases were selected among which 210 were found to have deranged lipid markers from normal value in human body(table1). The minimal age is 30 years and maximum age is 70 years. Most of the selected patients were in the age group of $46-55 \mathrm{yrs}(65 \%)$ followed by 56 70 yrs age group $(53 \%)$ as shown in graph 2 . There were 200 females and 183 male patients included in our study, among which 110 females (55\%) and 100 males (55\%) were found to have deranged lipid markers from normal value in human body graph 2 .

\section{DISCUSSION:}

This study has been conducted to see the association of deranged lipid profile in patients with diabetes mellitus. In our study it is observed that out of total 383 patients with diabetes mellitus, $210(55 \%)$ were found to have deranged lipid markers from normal value in human body. The results showed in our study that in diabetic patients the levels of different lipid markers were above the normal required range seen in human body and the outcome was also consistent with previous studies. ${ }^{17}$ In our study there were slightly more females 200, among which 55\% had deranged lipid markers from normal value in human body, as far as in males out of total 183 patients, $55 \%$ had deranged lipid markers from normal value in human body (graph1). The frequency of deranged lipid markers from normal value in human body was $55 \%$ in diabetic patients in our study is consistent with two different previous studies which also shows the presence of deranged lipid markers from normal value in human body in diabetic patients. ${ }^{18,19}$ Sedentary life style, increasing shift

\begin{tabular}{|c|c|c|}
\hline $\begin{array}{c}\text { Deranged lipid markers from } \\
\text { normal value in human body }\end{array}$ & Frequency & Percentage \\
\hline YES & 210 & $55 \%$ \\
\hline NO & 173 & $45 \%$ \\
\hline
\end{tabular}

Table: 1 frequency \& percentage of have deranged lipid markers from normal value

\begin{tabular}{|l|c|c|c|c|}
\hline Classification of lipids & Total cholesterol mg \% & LDL-C $\mathbf{~ m} \%$ & HDL -C $\mathbf{~ m g} \%$ & TG $\mathbf{~ m o} \%$ \\
\hline Desirable & $<200$ & $<100$ & $>60$ & $<150$ \\
\hline Near optimal & - & $100-129$ & Higher value is better & - \\
\hline Borderline High & $200-239$ & $130-159$ & - & $150-199$ \\
\hline High & 240 and above & $160-189$ & $59-40$ & $200-499$ \\
\hline Very high & - & 190 and above & $<40$ & 500 and above \\
\hline
\end{tabular}

Classification of lipids NCEP ATP 111 


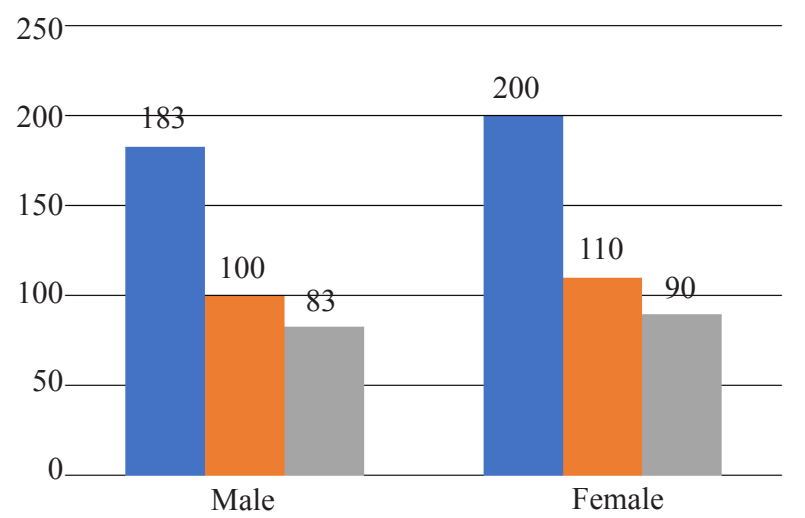

Total no of Patient $\square$ Dyslipidemic $\square$ No Dyslipidemia Graph 1: Distribution of deranged lipid markers from normal value in human body according to Gender

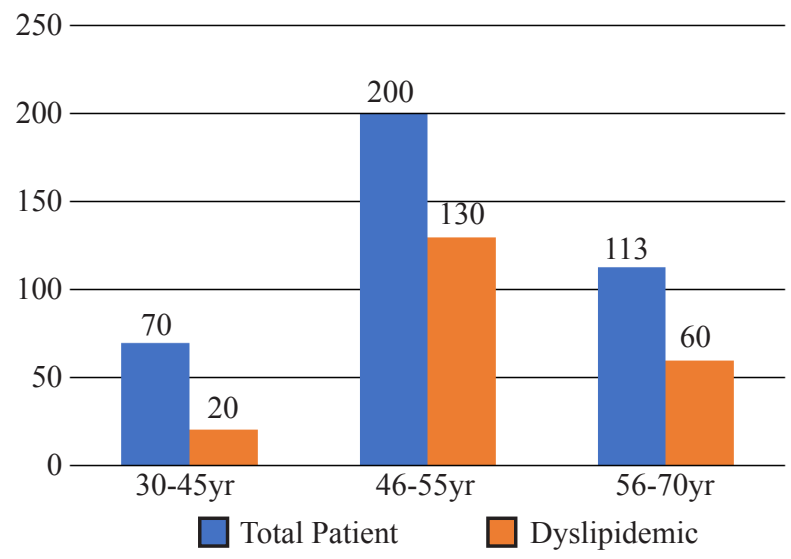

Graph 2: Distribution of deranged lipid markers from normal value in human body according to age group

\begin{tabular}{|l|l|c|}
\hline \multirow{2}{*}{$\begin{array}{l}\text { Total No of Patients with } \\
\text { deranged lipid markers } \\
\text { from normal value in } \\
\text { human body (210) }\end{array}$} & $\begin{array}{l}\text { No of patients with } \\
\text { elevated Serum } \\
\text { cholesterol } \\
140\end{array}$ & $\begin{array}{l}\text { Potal no of patients } \\
\text { with elevated Serum } \\
\text { triglycerides (TG) } \\
120\end{array}$ \\
\cline { 2 - 3 } & $\begin{array}{l}\text { Total no of patients } \\
\text { with elevated LDL- } \\
\text { C } \\
160\end{array}$ & $67 \%$ \\
\hline
\end{tabular}

Table 2: Percentage of Derangement in Lipid profile in Diabetics

of population from simple village life to mechanize life of cities, minimal physical activity due to the use of these hitech appliances and change of pure diet plan using junk meal causes increase weight that leads to development of tendency to have increase blood sugar level. ${ }^{20}$ The risk of cardiovascular diseases in diabetic patients is more likely as compared to normal human beings. Lipid abnormalities (increased level of low-density lipoprotein, triglycerides and decrease levels of High-density lipoprotein) are a predisposing factor for deposition of these lipid in arteries. Deranged lipid markers from normal value may be due to unbalanced metabolic state in diabetes and better control of diabetes does result in progressive decline in diabetesassociated deranged lipid markers from normal value in human body. In our study elevated low density lipoprotein (76\%) followed by the next common abnormalities being increase in serum cholesterol (66.6\%) and TG level. (57\%) (table 2) is also consistent with studies done in the past. ${ }^{21,22}$ In the other study done in past showed elevated triglycerides more than serum cholesterol. ${ }^{23}$ In our study maximum (68.5\%) of the sample population were 40 years. Comparing in different age groups, among diabetics, patients who are specially in 46 to 55 years of age, were more found to have deranged lipid markers from normal value in human body (65\%) (graph 2) then other age groups. The next age group which was more affected was $56-70$ years of age in which $53 \%$ of patients were having deranged lipid markers from normal value in human body (graph2). Previous studies also showed that increasing life span plays a major role in the risk of developing hyperglycemia. ${ }^{17,24}$ Mortality in diabetics is associated with deranged levels of different of lipid marker. ${ }^{19,25,26,27,28}$ So early detection and treatment of deranged lipid markers from normal value in human body especially with hyperglycemia can prevent the progression of disease and limit the morbidity and mortality due to cardiovascular events, and cerebrovascular accident. ${ }^{29}$ The limitation of the study was the small sample size in one lab and the financial constrains. It was recommended that patients with diabetes should be considered to be given lipid lowering therapy like statins along with life style modifications to prevent them from coronary artery disease especially in an age group of $55-70$ years.

\section{CONCLUSION:}

It was concluded that diabetes was associated with higher frequency of deranged lipid markers from normal value in human body and was associated with increased atherosclerotic changes in coronary arteries.

\section{REFRENCECES:}

1. Guariguata L, Whiting DR, Hambleton I, Beagley J, Linnenkamp U, Shaw JE. Global estimates of diabetes prevalence for 2013 and projections for 2035. Diabetes Research and Clinical Practice. 2014;103(2):137-49.

2. Maliha Sarfraz, Sanaullah Sajid, Muhammad Aqeel Ashraf. Prevalence and pattern of dyslipidemia in hyperglycemic patients and its associated factors among Pakistani population. Saudi Journal of Biological Sciences. 2016; 23: 761-66.

3. Abou-Seif MA, Youssef AA: Evaluation of some biochemical changes in diabetic patients. Clinica Chimica Acta; International journal of Clinical Chemistry. 2004; 346(2): 161-170.

4. Seshasai SR, Kaptoge S. Diabetes mellitus, fasting glucose, and risk of cause specific death. New England Journal of medicine .2011;364:829-41. 
5. Duckworth W, Araira C, Moritz T, Reda D. Glucose control and vascular complications in veterans with type 2 diabetes. New England journal of medicine.2009;360 (2):129-39.

6. Sarwar N, Gao P, Seshasai SR, Gobin Diabetes mellitus, fasting blood glucose concentration, and risk of vascular disease: a collaborative meta-analysis of 102 prospective studies. lancet.2010;375:2215-22.

7. Krishnaswami Vijayaraghavan. Treatment of dyslipidemia in patients with type 2 diabetes. Lipids in Health and Disease December 2010, 9(1):144

8. Taskinen MR. Diabetic dyslipidemia: from basic research to clinical practice. Diabetologia. 2003;46 (6):733-49.

9. Verges B. Pathophysiology of diabetic dyslipidemia: where are we? Diabetologia. 2015;58(5):886-99.

10. Donath MY, Schumann DM, Faulenbach M, Ellingsgaard H, Perren A, Ehses JA. Islet Inflammation in Type 2 Diabetes: from metabolic stress to therapy. Diabetes Care 2008;31(2): S161-S64.

11. Lee SJ, Campos H, Maye LA, Sacks FM. LDL containing apolipoprotein CIII is an independent risk factor for coronary events in diabetic patients. Arteriosclerosis, Thrombosis and Vascular Biology. 2003;23(5):853-8.

12. Abbasi A, Corpeleijn E, Gansevoort RT. Role of HDL cholesterol and estimates of HDL particle composition in future development of type 2 diabetes in general population: the PREVEND study. The Journal of clinical endocrinology and metabolism 2013;98(8):1352-59.

13. Modi KD, Chandwani R, Ahmed I, Kumar KV. Discordance between lipid markers used for predicting cardiovascular risk in patients with type 2 diabetes. Diabetes and Metabolic Syndrome. 2016;10(1):99-102.

14. Morgantini C, Natali A, Boldrini B. Anti-inflammatory and antioxidant properties of HDL are impaired in type 2 diabetes. Diabetes.2011;60 (10):2617-23.

15. Thapa Subarna Dhoj, KC Shiva Raj, Gautam Santosh, Gyawali Deepika. Dyslipidemia in type 2 diabetes mellitus. Journal of Pathology of Nepal 2017: 1149 - 54.

16. Nordestgaard BG, Varbo A. Triglycerides and cardiovascular disease. Lancet. 2014; 384:626-35.

17. Agrawal Y, Goyal V, Chugh K, Shanker, V., Singh AA. Types of dyslipidemia in type 2 diabetic patients of Haryana region. Scholars journal of Applied Medical Sciences.2014; 2(4): 1385-92.

18. Udawat H, Goyal RK, Maheshwari S. Coronary risk and dyslipidemia in type 2 diabetic patients. The Journal of Association of the Physicians of India. oct 2001;49: 970-73.
19. Jayarama N, Reddy M, Lakshmanan V. Prevalence and pattern of dyslipidemia in type 2 diabetes mellitus patients in a rural tertiary care Centre, South India. The Global Journal of Medicine and public health home 2012; 1(3): 24.

20. Bali K, Singh A. Pattern of dyslipidemia in Type 2 Diabetes Mellitus in Punjab. International Journal of Research in Medical Sciences.2016;4(3):809-12.

21. Ahmad A, Khan J, Siddiqui TS. Frequency of dyslipidemia in type 2 diabetes mellitus in patients of Hazara division. Journal of Ayub Medical College, Abbottabad 2008; 20 (2): $51-4$.

22. Kandula R, Shegokar VA. Study of lipid profile in patients with type 2 diabetes mellitus. Journal of Health Sciences. 1(1), 23-26.

23. Uttra KM, Devrajani BR, Shah SZA, Devrajani T, Das T, Raza S, Naseem. Lipid Profile of patients with Diabetes mellitus (a multidisciplinary study). World Applied Sciences Journal. 2011;12(9):1382-84.

24. Tagoe DNA, Amo-Kodieh P. Type 2 diabetes mellitus influences lipid profile of diabetic patients. Annals of Biological Research ,2013; 4(6): 88-92.

25. Nadeem A, Mumtaz Naveed AK et .al. Prevalence of dyslipidemia and impact of increasing age and duration of type 2 diabetes on dyslipidemia, insulin levels and insulin resistance. Jpma september 2015; 65(9)

26. M.Zaid, S.Hasnain . Plasma lipid abnormalities in Pakistani population: trends, associated factors, and clinical implications. Brazilian Journal of Medical Biological Research.2018; 51(9): e7239.

27. Muhammad Anwar1, Syed Raza Jaffar2, Aamir Ijaz3, Najamuddin1, Fawad Sana2.Evaluation of dyslipidaemia in type 2 diabetes mellitus patient. Pak journal of pathology. 2017: 28(3): 135-140.

28. McEneny J, O'Kane MJ, Moles KW, et al. Very low-density lipoprotein subfractions in type II diabetes mellitus: alterations in composition and susceptibility to oxidation. Diabetologia. 2000; 43:485-93.

29. Lotta LA, Sharp SJ, Burgess S, Perry JR, Stewart ID, Willems SM, Luan J, Ardanaz E, Arriola L, Balkau B. Association between low?density lipoprotein cholesterol?lowering genetic variants and risk of type 2 diabetes: a meta?analysis. JAMA. 2016;316(13):1383-91. 